\title{
Soft breaking of BRST symmetry and gauge dependence
}

\author{
P.M. Lavrov ${ }^{\dagger}$, O.V. Radchenko $^{\dagger}$ and A.A. Reshetnyak* \\ †Tomsk State Pedagogical University, \\ Kievskaya St. 60, 634061 Tomsk, Russia \\ * Institute of Strength Physics and Material Science, \\ Akademicheskii av. 2/4, 634021 Tomsk, Russia
}

\begin{abstract}
We continue investigation of soft breaking of BRST symmetry in the Batalin-Vilkovisky (BV) formalism beyond regularizations like dimensional ones used in our previous paper [JHEP 1110 (2011) 043, arXiv:1108.4820 [hep-th]]. We generalize a definition of soft breaking of BRST symmetry valid for general gauge theories and arbitrary gauge fixing. The gauge dependence of generating functionals of Green's functions is investigated. It is proved that such introduction of a soft breaking of BRST symmetry into gauge theories leads to inconsistency of the conventional BV formalism.
\end{abstract}

Emails: lavrov@tspu.edu.ru,radchenko@tspu.edu.ru,reshet@ispms.tsc.ru Keywords: Gauge theories, BRST symmetry, antibracket, field-antifield formalism PACS: $04.60 . \mathrm{Gw}, 11.30 . \mathrm{Pb}$ 


\section{Introduction}

A soft breaking of BRST symmetry in Yang-Mills theories has been intensively studied in a series of recent papers [1]. This breakdown is connected with a restriction of the domain of integration in the functional integral due to the Gribov horizon [2, 3, 4]. Note that all investigations $[1,3,4]$ of the Gribov horizon in Yang-Mills theories have been performed in the Landau gauge only. Some months ago a generalization of notation of a soft breaking of BRST symmetry for general gauge theories in arbitrary gauges in the framework of BV formalism [5] has been proposed in [6]. This approach to the problem of a soft breaking of BRST symmetry includes Yang-Mills theories in the Landau gauge as a very special case. In particular, it was shown that the way when one takes into account the Gribov horizon in the form of an additional term to the full action of given gauge system coinciding with Gribov-Zwanziger action $[2,3,4]$ in case of Yang-Mills theories in the Landau gauge leads to gauge dependent S-matrix.

In the present paper we extend the investigation performed in [6] to case when one uses any regularization scheme respecting gauge invariance.

The paper is organized as follows. In Section 2, our definition of the soft breaking of BRST symmetry is given in the BV formalism. In Section 3 we study different ways to introduce BRST-like transformations and derive the Ward identities for the generating functionals of Green's functions. In Section 4 we investigate the dependence of these functionals on gauges. Concluding remarks are given in Section 5.

We use the condensed notation of DeWitt [7]. Derivatives with respect to sources and antifields are taken from the left, while those with respect to fields are taken from the right. Left (right) derivatives with respect to fields (antifields) are labelled by a subscript $l(r)$. The Grassmann parity of any quantity $A$ in case of its homogeneity is denoted as $\varepsilon(A)$.

\section{Soft breaking of BRST symmetry in the BV formalism}

Consider a theory of gauge fields $A^{i}, i=1,2, \ldots, n,\left(\varepsilon\left(A^{i}\right)=\varepsilon_{i}\right)$, with an initial action $\mathcal{S}_{0}=\mathcal{S}_{0}(A)$. It is assumed the invariance of this action under the gauge transformations $\delta A^{i}=R_{\alpha}^{i}(A) \xi^{\alpha}$ with $\xi^{\alpha}\left(\varepsilon\left(\xi^{\alpha}\right)=\varepsilon_{\alpha}\right)$ to be arbitrary functions of the space-time coordinates that means the presence of the identities

$$
\mathcal{S}_{0, i}(A) R_{\alpha}^{i}(A)=0 \quad \text { for } \quad \alpha=1,2, \ldots, m, \quad 0<m<n
$$

among the classical extremals $\mathcal{S}_{0, i}=0$. Here, the functions $R_{\alpha}^{i}(A)\left(\varepsilon\left(R_{\alpha}^{i}\right)=\varepsilon_{i}+\varepsilon_{\alpha}\right)$ are the generators of the gauge transformations and we have used the notation $\mathcal{S}_{0, i} \equiv \delta \mathcal{S}_{0} / \delta A^{i}$. The structure of configuration space $\left\{\Phi^{A}\right\}$ in the BV formalism depends on the type of given classical gauge theory (for details, see [5]). For our purposes it is not important to describe its explicit 
contents. We need in the fact of existence of the total configuration space parameterized by the fields $\Phi \equiv\left\{\Phi^{A}\right\}=\left\{A^{i}, \ldots\right\}$ with $\varepsilon\left(\Phi^{A}\right)=\varepsilon_{A}$, where the dots indicate the full set of additional to $A^{i}$ fields in the BV method. Then to each field $\Phi^{A}$ of the configuration space, one should introduce the corresponding antifield $\Phi_{A}^{*}$ with opposite Grassmann parities to that of the corresponding field $\Phi^{A}, \Phi^{*} \equiv\left\{\Phi_{A}^{*}\right\}=\left\{A_{i}^{*}, \ldots\right\}$, with $\varepsilon\left(\Phi_{A}^{*}\right)=\varepsilon_{A}+1$.

The main object of the BV quantization is a bosonic functional $\bar{S}=\bar{S}\left(\Phi, \Phi^{*}\right)$ satisfying the master equation

$$
\frac{1}{2}(\bar{S}, \bar{S})=\mathrm{i} \hbar \Delta \bar{S}
$$

with the boundary condition

$$
\left.\bar{S}\right|_{\Phi^{*}=\hbar=0}=\mathcal{S}_{0}(A)
$$

Here we used the notation of odd (i.e. $\varepsilon(\Delta)=1$ ) nilpotent operator $\Delta$

$$
\Delta \equiv(-1)^{\varepsilon_{A}} \frac{\delta_{l}}{\delta \Phi^{A}} \frac{\delta}{\delta \Phi_{A}^{*}},
$$

and the antibracket, $(F, G)$, which can be reproduced by $\Delta$ acting on the product of two functionals $F$ and $G$ :

$$
\Delta F \cdot G=(\Delta F) \cdot G+F \cdot(\Delta G)(-1)^{\varepsilon(F)}+(F, G)^{\varepsilon(F)} .
$$

It is obvious that the initial action $\mathcal{S}_{0}$ satisfies the classical master equation, $\left(\mathcal{S}_{0}, \mathcal{S}_{0}\right)=0$.

Having the action $\bar{S}$ and a corresponding fermionic gauge fixing functional $\Psi=\Psi(\Phi)$, one can construct the non-degenerate action $S_{\text {ext }}$ by the rule

$$
S_{\text {ext }}\left(\Phi, \Phi^{*}\right)=\bar{S}\left(\Phi, \Phi^{*}+\frac{\delta \Psi}{\delta \Phi}\right)
$$

The action $S_{\text {ext }}$ satisfies the same master equation (2.2) as the functional $\bar{S}$,

$$
\frac{1}{2}\left(S_{\text {ext }}, S_{\text {ext }}\right)=\mathrm{i} \hbar \Delta S_{\text {ext }}
$$

and is used to construct the generating functional of Green's functions in the BV formalism [5].

Following to Refs. $[3,4,6]$, we deform the action $S_{\text {ext }}$ by adding a functional $M=M\left(\Phi, \Phi^{*}\right)$, thus determining the full action $S$ as

$$
S=S_{e x t}+M
$$

We shall speak of a soft breaking of BRST symmetry in the BV formalism if the condition

$$
\frac{1}{2}(M, M)=-\mathrm{i} \hbar \Delta M
$$


is fulfilled. Note, that in classical limit, $\hbar \rightarrow 0$, we assume that $M=M_{0}+O(\hbar)$, Eq. $(2.9)$ is reduced to

$$
\left(M_{0}, M_{0}\right)=0
$$

The reason to use the notation of "a soft breaking of BRST symmetry " may be explained as follows. The master equation (2.7) in the BV formalism can be presented in the form

$$
\Delta \exp \left\{\frac{\mathrm{i}}{\hbar} S_{\text {ext }}\right\}=0
$$

Using the action $S_{\text {ext }}$ as a solution to this equation in order to construct Green's functions for general gauge theories one can derive the BRST symmetry transformations [5]. Modifying the action $S_{\text {ext }}$ by a special functional $M$ (it allows us to speak of "soft") which satisfies the equation

$$
\Delta \exp \left\{-\frac{\mathrm{i}}{\hbar} M\right\}=0
$$

we obtain the action $S(2.8)$ not satisfying the equation likes (2.11)

$$
\Delta \exp \left\{\frac{\mathrm{i}}{\hbar} S\right\} \neq 0
$$

The BRST symmetry will be broken if we shall construct Green's functions in the BV formalism using this action (see Section 3 for details). From (2.7) and (2.9) it follows that the basic equation of our approach to the soft breaking of BRST symmetry reads

$$
\frac{1}{2}(S, S)-\mathrm{i} \hbar \Delta S=(S, M)
$$

In classical limit, for $S=S_{0}+O(\hbar)$, it follows from (2.14) the equation,

$$
\frac{1}{2}\left(S_{0}, S_{0}\right)=\left(S_{0}, M_{0}\right)
$$

coinciding in classical limit, $\hbar \rightarrow 0$, with the basic equation to the soft breaking of BRST symmetry considered in Ref.[6] when a regularization likes dimensional one for the local functional $S$ is applied, $\Delta S \sim \delta(0)=0$.

It is important to note that the condition (2.9) will be automatically valid in case when the soft breaking of BRST symmetry originates from a modification of the integration measure in the path integral. In this case $M$ will be a functional of the field variables $\Phi^{A}$ only, i.e. $M=$ $M(\Phi)$. As we have already mentioned in Ref. [6], this is exactly the situation for Yang-Mills theory in the Landau gauge, when one takes into account the Gribov horizon [3, 4]. We consider the more general situation of $M=M\left(\Phi, \Phi^{*}\right)$ not restricting ourselves to this special case.

Note that the right-hand side of the basic equation (2.14) can be presented in the form

$$
(S, M)=\hat{s} M-\mathrm{i} \hbar \Delta M
$$


where $\hat{s}$ denotes the quantum Slavnov-Taylor operator defined as $\hbar$-deformation of its classical analog in the form

$$
\hat{s}=\left(S_{e x t}, \bullet\right)-\mathrm{i} \hbar \Delta .
$$

Due to master equation for $S_{\text {ext }}(2.7)$ this operator is nilpotent,

$$
\hat{s}^{2}=0
$$

However, as compared to the consideration in [6], the presence of the additional term to $\hat{s}$ in the right-hand side of the relation (2.16) leads to the inequality

$$
\hat{s}\left\{\frac{1}{2}(S, S)-\mathrm{i} \hbar \Delta S\right\} \neq 0 \text {. }
$$

which being written for the action $S_{\text {ext }}$ should be the identity for general gauge theories without a (soft) breaking of BRST symmetry.

\section{BRST transformations and Ward identities}

Let us consider some quantum consequences of the classical equations (2.7), (2.9) and (2.14). To this end we introduce the generating functional of Green's functions,

$$
Z\left(J, \Phi^{*}\right)=\int D \Phi \exp \left\{\frac{\mathrm{i}}{\hbar}\left(S\left(\Phi, \Phi^{*}\right)+J_{A} \Phi^{A}\right)\right\}
$$

where $S\left(\Phi, \Phi^{*}\right)$ satisfies the basic classical equation (2.14) and has the form (2.8). Furthermore, $J_{A}$ are the usual external sources for the fields $\Phi^{A}$. The Grassmann parities of these sources are defined in a natural way, $\varepsilon\left(J_{A}\right)=\varepsilon_{A}$.

Let us consider the vacuum functional $Z\left(0, \Phi^{*}\right)=Z\left(\Phi^{*}\right)$ and its integrand

$$
\mathcal{M}=\mathcal{M}\left(\Phi, \Phi^{*}\right)=D \Phi \exp \left\{\frac{\mathrm{i}}{\hbar} S\left(\Phi, \Phi^{*}\right)\right\} .
$$

In the BV formalism the BRST symmetry appears as invariance of the integrand of vacuum functional under the change of variables, $\Phi^{A} \rightarrow \Phi^{\prime A}=\Phi^{A}+\delta_{B} \Phi^{A}$ determined with the help of $S_{\text {ext }}\left(\Phi, \Phi^{*}\right)$,

$$
\delta_{B} \Phi^{A}=\frac{\delta S_{e x t}}{\delta \Phi_{A}^{*}} \theta, \quad \delta_{B} \Phi_{A}^{*}=0
$$

where $\theta$ is a nilpotent constant odd parameter. Carrying out the change of variables (3.3) in (3.2) we obtain

$$
\mathcal{M}^{\prime}=\mathcal{M}\left(1-\frac{\mathrm{i}}{\hbar} \theta \frac{\delta M}{\delta \Phi^{A}} \frac{\delta S_{e x t}}{\delta \Phi_{A}^{*}}\right)
$$


Non-invariance of the integrand means violation of the standard BRST symmetry. Of course, one may think that it is necessary to modify the definition of BRST transformations in case of the theory under consideration. A possible way for its modification looks as

$$
\delta_{B} \Phi^{A}=\frac{\delta S}{\delta \Phi_{A}^{*}} \theta, \quad \delta_{B} \Phi_{A}^{*}=0,
$$

Performing the change of variables (3.5) in the integrand functional $\mathcal{M}(3.2)$ one has

$$
\mathcal{M}^{\prime}=\mathcal{M}\left(1-\frac{\mathrm{i}}{\hbar} \theta(S, M)\right)
$$

We meet again the non-invariance under modified BRST transformations (3.5). Moreover one can consider the following one-parameter functional $S_{\kappa}$

$$
S_{\kappa}=S_{e x t}+\kappa M
$$

to define the BRST trasformations

$$
\delta_{B_{\kappa}} \Phi^{A}=\frac{\delta S_{\kappa}}{\delta \Phi_{A}^{*}} \theta, \quad \delta_{B_{\kappa}} \Phi_{A}^{*}=0 .
$$

Here $\kappa$ is a real number. We have the standard BRST transformations (3.3) for $\kappa=0$ and the modified ones (3.5) for $\kappa=1$. Carrying out the change of variables (3.8) in the integrand (3.2) we arrive at the result

$$
\mathcal{M}^{\prime}=\mathcal{M}\left(1-\frac{\mathrm{i}}{\hbar} \theta\left[\kappa\left(S_{\text {ext }}, M\right)-2 \mathrm{i} \hbar \kappa \Delta M+(1-\kappa) \frac{\delta M}{\delta \Phi^{A}} \frac{\delta S_{\text {ext }}}{\delta \Phi_{A}^{*}}\right]\right) .
$$

We may conclude that the non-invariance of the integrand exists for any choice of the parameter $\kappa$.

Turning to the properties of the generating functional $Z\left(J, \Phi^{*}\right)$ note, that from the Eq. (2.11) it follows that its averaging over the total configuration space of the fields $\Phi^{A}$ with measure $\exp \left\{\frac{\mathrm{i}}{\hbar}\left(S+J_{A} \Phi^{A}\right)\right\}$

$$
0=\int D \Phi \Delta \exp \left\{\frac{\mathrm{i}}{\hbar} S_{\text {ext }}\right\} \exp \left\{\frac{\mathrm{i}}{\hbar}\left(S+J_{A} \Phi^{A}\right)\right\}
$$

leads after integrating by parts in the functional integral to the following identity for the generating functional $Z$,

$$
\left(J_{A}+M_{A}\left(\frac{\hbar}{\mathrm{i}} \frac{\delta}{\delta J}, \Phi^{*}\right)\right)\left(\frac{\hbar}{\mathrm{i}} \frac{\delta}{\delta \Phi_{A}^{*}}-M^{A *}\left(\frac{\hbar}{\mathrm{i}} \frac{\delta}{\delta J}, \Phi^{*}\right)\right) Z\left(J, \Phi^{*}\right)=0 .
$$

Here the notations

$$
\left.M_{A}\left(\frac{\hbar}{\mathrm{i}} \frac{\delta}{\delta J}, \Phi^{*}\right) \equiv \frac{\delta M\left(\Phi, \Phi^{*}\right)}{\delta \Phi^{A}}\right|_{\Phi \rightarrow \frac{\hbar}{\mathrm{i}} \frac{\delta}{\delta J}} \quad \text { and }\left.\quad M^{A *}\left(\frac{\hbar}{\mathrm{i}} \frac{\delta}{\delta J}, \Phi^{*}\right) \equiv \frac{\delta M\left(\Phi, \Phi^{*}\right)}{\delta \Phi_{A}^{*}}\right|_{\Phi \rightarrow \frac{\hbar}{\mathrm{i}} \frac{\delta}{\delta J}}
$$


have been used. In case of $M=0$, the identity (3.10) is reduced to the usual Ward identity for the generating functional of Green's functions in the BV formalism. Hence, we refer to (3.10) as the Ward identity for $Z$ in a gauge theory with softly broken BRST symmetry. Note, that for the regularization scheme likes dimensional one, we will have $\Delta M=0$ and, therefore the equation (2.9) is reduced to $(M, M)=0$, which leads to the vanishing of the combination, $M_{A}\left(\frac{\hbar}{\mathrm{i}} \frac{\delta}{\delta J}, \Phi^{*}\right) M^{A *}\left(\frac{\hbar}{\mathrm{i}} \frac{\delta}{\delta J}, \Phi^{*}\right)$ in the Ward identity (3.10) as it was derived in [6].

Then, introducing the generating functional of connected Green's functions,

$$
W\left(J, \Phi^{*}\right)=-\mathrm{i} \hbar \ln Z\left(J, \Phi^{*}\right),
$$

the identity (3.10) can be rewritten for $W$ as

$$
\left(J_{A}+M_{A}\left(\frac{\delta W}{\delta J}+\frac{\hbar}{\mathrm{i}} \frac{\delta}{\delta J}, \Phi^{*}\right)\right)\left(\frac{\delta W\left(J, \Phi^{*}\right)}{\delta \Phi_{A}^{*}}-M^{A *}\left(\frac{\delta W}{\delta J}+\frac{\hbar}{\mathrm{i}} \frac{\delta}{\delta J}, \Phi^{*}\right)\right)=0 .
$$

The generating functional of the vertex functions (or effective action) is obtained by Legendre transforming of $W$,

$$
\Gamma\left(\Phi, \Phi^{*}\right)=W\left(J, \Phi^{*}\right)-J_{A} \Phi^{A}, \quad \text { where } \quad \Phi^{A}=\frac{\delta W}{\delta J_{A}}, \quad \frac{\delta \Gamma}{\delta \Phi^{A}}=-J_{A} .
$$

Taking into account the equality, $\frac{\delta \Gamma}{\delta \Phi_{A}^{*}}=\frac{\delta W}{\delta \Phi_{A}^{*}}$, we can rewrite the identity (3.12) in terms of $\Gamma$ as

$$
\frac{1}{2}(\Gamma, \Gamma)=\frac{\delta \Gamma}{\delta \Phi^{A}} \widehat{M}^{A *}+\widehat{M}_{A} \frac{\delta \Gamma}{\delta \Phi_{A}^{*}}-\widehat{M}_{A} \widehat{M}^{A *}
$$

Here, we have used the notations

$$
\left.\widehat{M}_{A} \equiv \frac{\delta M\left(\Phi, \Phi^{*}\right)}{\delta \Phi^{A}}\right|_{\Phi \rightarrow \widehat{\Phi}} \quad \text { and }\left.\quad \widehat{M}^{A *} \equiv \frac{\delta M\left(\Phi, \Phi^{*}\right)}{\delta \Phi_{A}^{*}}\right|_{\Phi \rightarrow \widehat{\Phi}^{\prime}},
$$

where the sign $\widehat{\Phi}^{A}$ means the field $\Phi^{A}$ enlarged by the derivatives $\frac{\delta_{l}}{\delta \Phi^{B}}$,

$$
\widehat{\Phi}^{A}=\Phi^{A}+\mathrm{i} \hbar\left(\Gamma^{\prime \prime}-1\right)^{A B} \frac{\delta_{l}}{\delta \Phi^{B}}
$$

and the matrix $\left(\Gamma^{\prime \prime}-1\right)$ is inverse to the matrix $\Gamma^{\prime \prime}$ with elements

$$
\left(\Gamma^{\prime \prime}\right)_{A B}=\frac{\delta_{l}}{\delta \Phi^{A}}\left(\frac{\delta \Gamma}{\delta \Phi^{B}}\right), \quad \text { i.e. } \quad\left(\Gamma^{\prime \prime}-1\right)^{A C}\left(\Gamma^{\prime \prime}\right)_{C B}=\delta_{B}^{A} .
$$

We see again, in the case $M=0$ the identity (3.14) coincides with the Ward identity for the effective action in the BV formalism. Emphasize that the identity (3.14) is compatible with the classical equation (2.14), since $\hbar \rightarrow 0$ yields $\Gamma=S_{0}, \widehat{M}=M_{0}$, and (3.14) is reduced to (2.15).

In similar manner we can derive the Ward identity which follows from (2.9). To this end, we average the equation (2.9) over the configuration space of the fields $\Phi^{A}$ with measure $\exp \left\{\frac{\mathrm{i}}{\hbar}(S+\right.$ $\left.\left.J_{A} \Phi^{A}\right)\right\}$,

$$
0=\int D \Phi\left\{\frac{\delta M}{\delta \Phi^{A}} \frac{\delta M}{\delta \Phi_{A}^{*}}+\mathrm{i} \hbar \Delta M\right\} \exp \left\{\frac{\mathrm{i}}{\hbar}\left(S+J_{A} \Phi^{A}\right)\right\}
$$


and derive after usual manipulations with functional integral the identity in terms of mean fields $\Phi^{A}(3.13)$

$$
\widehat{M}_{A} \widehat{M}^{A *}=-i \hbar \widehat{M}_{A}^{A^{*}}
$$

where the notation

$$
\widehat{M}_{A}^{A^{*}}=\left.\frac{\delta^{2} M}{\delta \Phi_{A}^{*} \delta \Phi^{A}}\right|_{\Phi \rightarrow \widehat{\Phi}}
$$

was used. The identity (3.18) is reduced to the known identity, $\widehat{M}_{A} \widehat{M}^{A *}=0$, derived in [6], when the regularization scheme likes dimensional one is applied.

\section{Gauge dependence}

Here we study the gauge dependence of the generating functionals $Z, W$ and $\Gamma$ for general gauge theories with a soft breaking of BRST symmetry as it was defined in the section above. The derivation of this dependence is based on the fact that any variation of the gauge-fixing functional, $\Psi(\Phi) \rightarrow \Psi(\Phi)+\delta \Psi(\Phi)$, leads to a variation both the action $S_{\text {ext }}(2.6)$, the functional $Z$ [8] and the functional $M$. The variation of $S_{\text {ext }}$ can be presented in the form

$$
\delta S_{e x t}=\frac{\delta \delta \Psi}{\delta \Phi^{A}} \frac{\delta S_{e x t}}{\delta \Phi_{A}^{*}} \quad \text { or as } \quad \delta S_{e x t}=-\left(S_{e x t}, \delta \Psi\right)=-\hat{s} \delta \Psi,
$$

whereas the variation of $M$ for the variation $\delta \Psi$ we denote as $\delta M\left(\Phi, \Phi^{*}\right)$. From (3.1), (4.1) and the variation of $M$ we obtain the gauge variation of $Z$,

$$
\delta Z\left(J, \Phi^{*}\right)=\frac{\mathrm{i}}{\hbar} \int D \Phi\left(\frac{\delta \delta \Psi}{\delta \Phi^{A}} \frac{\delta S_{\text {ext }}}{\delta \Phi_{A}^{*}}+\delta M\right) \exp \left\{\frac{\mathrm{i}}{\hbar}\left(S\left(\Phi, \Phi^{*}\right)+J_{A} \Phi^{A}\right)\right\} .
$$

Then applying the equality

$$
\begin{aligned}
0 & =\int D \Phi \frac{\delta_{l}}{\delta \Phi^{A}}\left[\delta \Psi \frac{\delta S_{\text {ext }}}{\delta \Phi_{A}^{*}} \exp \left\{\frac{\mathrm{i}}{\hbar}\left(S\left(\Phi, \Phi^{*}\right)+J_{A} \Phi^{A}\right)\right\}\right] \\
& =\int D \Phi\left[\frac{\delta \delta \Psi}{\delta \Phi^{A}} \frac{\delta S_{\text {ext }}}{\delta \Phi_{A}^{*}}-\frac{\mathrm{i}}{\hbar}\left(J_{A}+\frac{\delta S}{\delta \Phi^{A}}\right) \frac{\delta S_{\text {ext }}}{\delta \Phi_{A}^{*}} \delta \Psi\right] \exp \left\{\frac{\mathrm{i}}{\hbar}\left(S\left(\Phi, \Phi^{*}\right)+J_{A} \Phi^{A}\right)\right\}
\end{aligned}
$$

for which validity the equation (2.7) was used, we can rewrite (4.2) as

$$
\begin{aligned}
\delta Z\left(J, \Phi^{*}\right)= & \frac{\mathrm{i}}{\hbar}\left[\left(J_{A}+M_{A}\left(\frac{\hbar}{\mathrm{i}} \frac{\delta}{\delta J}, \Phi^{*}\right)\right)\left(\frac{\delta}{\delta \Phi_{A}^{*}}-\frac{\mathrm{i}}{\hbar} M^{A *}\left(\frac{\hbar}{\mathrm{i}} \frac{\delta}{\delta J}, \Phi^{*}\right)\right) \delta \Psi\left(\frac{\hbar}{\mathrm{i}} \frac{\delta}{\delta J}\right)\right. \\
& \left.+\delta M\left(\frac{\hbar}{\mathrm{i}} \frac{\delta}{\delta J}, \Phi^{*}\right)\right] Z\left(J, \Phi^{*}\right) .
\end{aligned}
$$

In turn, the corresponding variation of the generating functional of connected Green's functions, $\delta W\left(J, \Phi^{*}\right)=\frac{\hbar}{\mathrm{i}} Z^{-1} \delta Z$, takes the form

$$
\delta W\left(J, \Phi^{*}\right)=\left(J_{A}+M_{A}\left(\frac{\delta W}{\delta J}+\frac{\hbar}{\mathrm{i}} \frac{\delta}{\delta J}, \Phi^{*}\right)\right) \frac{\delta}{\delta \Phi_{A}^{*}} \delta \Psi\left(\frac{\delta W}{\delta J}+\frac{\hbar}{\mathrm{i}} \frac{\delta}{\delta J}\right)+\delta M\left(\frac{\delta W}{\delta J}+\frac{\hbar}{\mathrm{i}} \frac{\delta}{\delta J}, \Phi^{*}\right),
$$


with using the Ward identity (3.12).

Now, we are able to arrive at our final purpose concerning the derivation of the gauge variation of the effective action. First, we note that $\delta \Gamma=\delta W$. Second, we observe that the change of the variables $\left(J_{A}, \Phi_{A}^{*}\right) \rightarrow\left(\Phi^{A}, \Phi_{A}^{*}\right)$ from the Legendre transformation (3.13) implies that

$$
\left.\frac{\delta}{\delta \Phi^{*}}\right|_{J}=\left.\frac{\delta}{\delta \Phi^{*}}\right|_{\Phi}+\left.\frac{\delta \Phi}{\delta \Phi^{*}} \frac{\delta_{l}}{\delta \Phi}\right|_{\Phi^{*}}
$$

Next, differentiating the Ward identities for $Z$ (3.10) with respect to the sources $J_{B}$, we obtain

$$
\begin{aligned}
& \frac{\hbar}{i} \frac{\delta Z}{\delta \Phi_{B}^{*}}+\frac{\hbar}{i}\left(J_{A}+M_{A}\left(\frac{\hbar}{\mathrm{i}} \frac{\delta}{\delta J}, \Phi^{*}\right) \frac{\delta^{2} Z}{\delta J_{B} \delta \Phi_{A}^{*}}(-1)^{\varepsilon_{A} \varepsilon_{B}}-M^{B *}\left(\frac{\hbar}{\mathrm{i}} \frac{\delta}{\delta J}, \Phi^{*}\right) Z-\right. \\
& -(-1)^{\varepsilon_{B}} J_{A} M^{A *}\left(\frac{\hbar}{\mathrm{i}} \frac{\delta}{\delta J}, \Phi^{*}\right) \frac{\delta Z}{\delta J_{B}}-(-1)^{\varepsilon_{B}} M_{A}\left(\frac{\hbar}{\mathrm{i}} \frac{\delta}{\delta J}, \Phi^{*}\right) M^{A *}\left(\frac{\hbar}{\mathrm{i}} \frac{\delta}{\delta J}, \Phi^{*}\right) \frac{\delta Z}{\delta J_{B}}=0 .
\end{aligned}
$$

Using the interrelation of the derivatives for $Z$ and $W$,

$$
\begin{aligned}
& \left(\frac{\delta Z}{\delta J_{A}}, \frac{\delta Z}{\delta \Phi_{A}^{*}}\right)=\frac{\mathrm{i}}{\hbar} \exp \left\{\frac{\mathrm{i}}{\hbar} W\right\}\left(\frac{\delta W}{\delta J_{A}}, \frac{\delta W}{\delta \Phi_{A}^{*}}\right), \\
& \frac{\delta^{2} Z}{\delta \Phi_{B}^{*} \delta J_{A}}=\exp \left\{\frac{\mathrm{i}}{\hbar} W\right\}\left[\left(\frac{\mathrm{i}}{\hbar}\right)^{2} \frac{\delta W}{\delta \Phi_{B}^{*}} \frac{\delta W}{\delta J_{A}}+\frac{\mathrm{i}}{\hbar} \frac{\delta^{2} W}{\delta \Phi_{B}^{*} \delta J_{A}}\right]
\end{aligned}
$$

the Eqs. (4.6) may be written in terms of functional $W$ as,

$$
\begin{aligned}
& \frac{\delta W\left(J, \Phi^{*}\right)}{\delta \Phi_{B}^{*}}+(-1)^{\varepsilon_{B}}\left(J_{A}+M_{A}\left(\frac{\hbar}{\mathrm{i}} \frac{\delta}{\delta J}+\frac{\delta W}{\delta J}, \Phi^{*}\right)\right) \frac{\delta^{2} W\left(J, \Phi^{*}\right)}{\delta \Phi_{A}^{*} \delta J_{B}}-M^{B *}\left(\frac{\hbar}{\mathrm{i}} \frac{\delta}{\delta J}+\frac{\delta W}{\delta J}, \Phi^{*}\right) \\
& =-\frac{\mathrm{i}}{\hbar}(-1)^{\varepsilon_{B}}\left(J_{A}+M_{A}\left(\frac{\hbar}{\mathrm{i}} \frac{\delta}{\delta J}+\frac{\delta W}{\delta J}, \Phi^{*}\right)\right)\left(\frac{\delta W}{\delta \Phi_{A}^{*}}-M^{A *}\left(\frac{\hbar}{\mathrm{i}} \frac{\delta}{\delta J}+\frac{\delta W}{\delta J}, \Phi^{*}\right)\right) \frac{\delta W}{\delta J_{B}} .
\end{aligned}
$$

From (4.9) it follows

$$
\frac{\delta \Gamma}{\delta \Phi_{B}^{*}}-\widehat{M}^{B^{*}}-\left(\frac{\delta \Gamma}{\delta \Phi^{A}}-\widehat{M}_{A}\right) \frac{\delta \Phi^{B}}{\delta \Phi_{A}^{*}}(-1)^{\varepsilon_{B}}=\frac{i}{\hbar}(-1)^{\varepsilon_{B}}\left(\frac{\delta \Gamma}{\delta \Phi^{A}}-\widehat{M}_{A}\right)\left(\frac{\delta \Gamma}{\delta \Phi_{A}^{*}}-\widehat{M}^{A^{*}}\right) \Phi^{B}
$$

To simplify the above expression one should commute the fields $\Phi^{B}$ to the left in the last summand in order to use Ward identity for effective action $\Gamma$ (3.14) As a result, we rewrite the relation $(4.10)$ in the form

$$
\begin{aligned}
-\left(\frac{\delta \Gamma}{\delta \Phi^{A}}-\widehat{M}_{A}\right) \frac{\delta \Phi^{B}}{\delta \Phi_{A}^{*}}= & -\left(\frac{\delta \Gamma}{\delta \Phi_{B}^{*}}-\widehat{M}^{B^{*}}\right)(-1)^{\varepsilon_{B}} \\
& +\frac{i}{\hbar}\left[-\widehat{M}_{A} \frac{\delta \Gamma}{\delta \Phi_{A}^{*}}-\frac{\delta \Gamma}{\delta \Phi^{A}} \widehat{M}^{A^{*}}+\widehat{M}_{A} \widehat{M}^{A^{*}}, \Phi^{B}\right],
\end{aligned}
$$

where the square brackets $[$,$] denote the supercommutator.$ 
From (4.4), (4.5) and (4.11) the variation of the effective action can be presented in the form,

$$
\begin{aligned}
\delta \Gamma= & -(\Gamma,\langle\delta \Psi\rangle)+\left(\widehat{M}_{A} \frac{\delta}{\delta \Phi_{A}^{*}}+(-1)^{\varepsilon_{A}} \widehat{M}^{A *} \frac{\delta_{l}}{\delta \Phi^{A}}\right)\langle\delta \Psi\rangle \\
& -\frac{\mathrm{i}}{\hbar}\left[\widehat{M}_{A} \frac{\delta \Gamma}{\delta \Phi_{A}^{*}}+\frac{\delta \Gamma}{\delta \Phi^{A}} \widehat{M}^{A *}-\widehat{M}_{A} \widehat{M}^{A *}, \Phi^{B}\right] \frac{\delta_{l}}{\delta \Phi^{B}}\langle\delta \Psi\rangle+\langle\delta M\rangle,
\end{aligned}
$$

with local (for $M=0$ ) operator acting on the functional $\langle\delta \Psi\rangle$. Here we imply the notations

$$
\langle\delta \Psi\rangle=\delta \Psi(\widehat{\Phi}) \cdot 1 \quad \text { and } \quad\langle\delta M\rangle=\delta M\left(\widehat{\Phi}, \Phi^{*}\right) \cdot 1 .
$$

Then, using the identities,

$$
\frac{\delta \Phi^{B}}{\delta \Phi_{A}^{*}}=(-1)^{\varepsilon_{B}\left(\varepsilon_{A}+1\right)} \frac{\delta}{\delta J_{B}} \frac{\delta W}{\delta \Phi_{A}^{*}}=-(-1)^{\varepsilon_{B}\left(\varepsilon_{A}+1\right)}\left(\Gamma^{\prime \prime}-1\right)^{B C} \frac{\delta_{l}}{\delta \Phi^{C}} \frac{\delta \Gamma}{\delta \Phi_{A}^{*}},
$$

following from the Legendre transformation (3.13) we can present the variation of the effective action in the equivalent, so-called non-local (due to explicit presence of the quantities $\left(\Gamma^{\prime \prime}-1\right)^{B C}$ ) form,

$$
\delta \Gamma=\frac{\delta \Gamma}{\delta \Phi^{A}} \widehat{F}^{A}\langle\delta \Psi\rangle-\widehat{M}_{A} \widehat{F}^{A}\langle\delta \Psi\rangle+\langle\delta M\rangle,
$$

where the operator $\widehat{F}^{A}$ is derived from the Eqs. (4.5), (4.11), (4.12) as follows

$$
\widehat{F}^{A}=-\frac{\delta}{\delta \Phi_{A}^{*}}+(-1)^{\varepsilon_{B}\left(\varepsilon_{A}+1\right)}\left(\Gamma^{\prime \prime}-1\right)^{B C}\left(\frac{\delta_{l}}{\delta \Phi^{C}} \frac{\delta \Gamma}{\delta \Phi_{A}^{*}}\right) \frac{\delta_{l}}{\delta \Phi^{B}} .
$$

From the variation (4.15) it follows that on shell the effective action is generally gauge dependent because of

$$
\frac{\delta \Gamma}{\delta \Phi^{A}}=0 \quad \longrightarrow \quad \delta \Gamma \neq 0
$$

This fact does not permit to formulate consistently a soft breaking of BRST symmetry within the field-antifield formalism, if only two last terms in (4.15) cancel each other,

$$
\langle\delta M\rangle=\widehat{M}_{A} \widehat{F}^{A}\langle\delta \Psi\rangle
$$

However, this is rather a strong restriction on the BRST-breaking functional $M$ for the effective action to be gauge independent on-shell. The same statement is valid for the physical S-matrix. Really, Eq. (4.18) fixes the gauge variation of $M=M\left(\Phi, \Phi^{*}\right)$ under a change of the gauge-fixing functional $\Psi$ to be

$$
\delta M=\frac{\delta M}{\delta \Phi^{A}} \widehat{F}_{0}^{A} \delta \Psi \quad \text { where } \quad \widehat{F}_{0}^{A}=(-1)^{\varepsilon_{B}\left(\varepsilon_{A}+1\right)}\left(S^{\prime \prime}-1\right)^{B C}\left(\frac{\delta_{l}}{\delta \Phi^{C}} \frac{\delta S}{\delta \Phi_{A}^{*}}\right) \frac{\delta_{l}}{\delta \Phi^{B}} .
$$

It was shown in [6] that already in the case of Yang-Mills theories in linear $R_{\xi}$ gauge which includes the Landau gauge the relation (4.19) does not satisfy. We are forced to claim that a consistent quantization of general gauge theories when restriction on the domain of integration in functional integral is taken as an addition to the full action of a given gauge system violating the BRST symmetry does not exist. 


\section{Conclusions}

In the given paper we have suggested a definition of soft breaking of BRST symmetry in the BV formalism using any regularization scheme respecting gauge invariance. To this purpose, we added a 'breaking functional' $M$ to the gauge-fixed action $S_{\text {ext }}$ which, in turn, is constructed from an arbitrary classical gauge-invariant action $\mathcal{S}_{0}$ according to the rules of the BV method. The soft breaking of BRST symmetry was determined by the analog of the quantum master equation, $(M, M)=-2 i \hbar \Delta M$. It was proved the non-invariance of the integrand of vacuum functional under the BRST transformations determined by means of the functional, $\left(S_{\text {ext }}+\kappa M\right)$, for any value of the real parameter $\kappa$. We have obtained all Ward identities for the generating functional of Green's functions $Z$, of connected Green's functions $W$ and of vertex functions $\Gamma$ being different from ones in Ref.[6] due to non-vanishing, in general, of the operator $\Delta$ action on the functionals $S_{\text {ext }}$ and $M$. The Ward identities were used to investigate the gauge dependence of those functionals. It was argued that $\Gamma$ as well as the S-matrix are on-shell gauge dependent. We were forced to claim that a consistent quantization of gauge systems in the BV formalism with the soft breaking of BRST symmetry does not exist.

\section{Acknowledgments}

The work is supported by the LRSS grant 224.2012.2 as well as by the RFBR grant 12-02-00121 and the RFBR-Ukraine grant 11-02-90445.

\section{References}

[1] M.A.L. Capri, A.J. Gómes, M.S. Guimaraes, V.E.R. Lemes, S.P. Sorellao and D.G. Tedesko, A remark on the BRST symmetry in the Gribov-Zwanzider theory, Phys. Rev. D82 (2010) 105019, arXiv:1009.4135 [hep-th];

L. Baulieu, M.A.L. Capri, A.J. Gomes, M.S. Guimaraes, V.E.R. Lemes, R.F. Sobreiro and S.P. Sorella, Renormalizability of a quark-gluon model with soft BRST breaking in the infrared region, Eur. Phys. J. C66 (2010) 451, arXiv:0901.3158 [hep-th];

D. Dudal, S.P. Sorella, N. Vandersickel and H. Verschelde, Gribov no-pole condition, Zwanziger horizon function, Kugo-Ojima confinement criterion, boundary conditions, BRST breaking and all that, Phys. Rev. D79 (2009) 121701, arXiv:0904.0641 [hep-th];

L. Baulieu and S.P. Sorella, Soft breaking of BRST invariance for introducing nonperturbative infrared effects in a local and renormalizable way,

Phys. Lett. B671 (2009) 481, arXiv:0808.1356 [hep-th];

M.A.L. Capri, A.J. Gómes, M.S. Guimaraes, V.E.R. Lemes, S.P. Sorella and D.G. Tedesko, Renormalizability of the linearly broken formulation of the BRST symmetry in presence of 
the Gribov horizon in Landau gauge Euclidean Yang-Mills theories, arXiv:1102.5695 [hep-th];

D. Dudal, S.P. Sorella and N. Vandersickel, The dynamical origin of the refinement of the Gribov-Zwanziger theory, arXiv:1105.3371 [hep-th].

R.F. Sobreiro and S.P. Sorella, A study of the Gribov copies in linear covariant gauges in Euclidean Yang-Mills theories, JHEP 0506 (2005) 054, arXiv:hep-th/0506165.

[2] V.N. Gribov, Quantization of nonabelian gauge theories, Nucl.Phys. B139 (1978) 1.

[3] D. Zwanziger, Action from the Gribov horizon, Nucl. Phys. B321 (1989) 591.

[4] D. Zwanziger, Local and renormalizable action from the Gribov horizon, Nucl. Phys. B323 (1989) 513.

[5] I.A. Batalin and G.A. Vilkovisky, Gauge algebra and quantization, Phys. Lett. 102B (1981) 27;

I.A. Batalin and G.A. Vilkovisky, Quantization of gauge theories with linearly dependent generators, Phys. Rev. D28 (1983) 2567.

[6] P. Lavrov, O. Lechtenfeld and A. Reshetnyak, Is soft breaking of BRST symmetry consistent?, JHEP 1110 (2011) 043, arXiv:1108.4820 [hep-th].

[7] B.S. DeWitt, Dynamical theory of groups and fields, Gordon and Breach, 1965.

[8] B.L. Voronov, P.M. Lavrov and I.V. Tyutin, Canonical transformations and gauge dependence in general gauge theories, Sov. J. Nucl. Phys. 36 (1982) 292. 